\title{
Classification of Micro Calcifications in Mammogram using Combined Feature Set with SVM
}

\author{
Prof.Dr.C.Manoharan \\ Principal, VSA Groups of Educational Institutions
}

\author{
N.V.S.Sree Rathna Lakshmi \\ Panimalar Institue of Technology
}

\begin{abstract}
Mammography is the only effective and viable technique to detect breast cancer especially in the case of minimal tumors. About $30 \%$ to $50 \%$ of breast cancers demonstrate deposits of calcium called micro calcifications. Our method proposes an approach for detecting microcalcification in mammograms based on combined feature set with Support Vector Machine (SVM) classifier. The diagonal matrix 'S' obtained from the Singular Value Decomposition (SVD) of LL band of wavelet transform is used as one of feature set for the classification of mammogram The set of Jacobi polynomials are orthogonal and this ensures minimal information redundancy between the moments. Jacobi moments encompass the properties of well known Zernike, Legendre and Tchebichef moments. Thus Jacobi moments are used combined with ' $\mathrm{S}$ ' matrix to achieve the better classification result.
\end{abstract}

Keywords: Jacobi Polynomials, Jacobi moments, SVD, SVM, micro calcifications

\section{INTRODUCTION}

Intensive research work has been undertaken in the development of automated image analysis methods to assist radiologists in the identification of abnormalities. The role computers play in mammogram analysis is threefold: detection, diagnosis and noise cancellation [1]. Detection involves identifying cancerous tissues in a mammogram. Early detection of breast cancer by mammography depends on the production of excellent images and competent interpretation. Mammography alone cannot prove that a suspicious area is malignant or benign. To decide that, the tissue has to be removed for examination using breast biopsy techniques. Diagnosis using mammograms is aimed at classifying the detected cancerous regions as benign or malignant.

Many studies have been made on the problem of breast cancer diagnosing based on digital mammograms. Different methods have been used to classify and/or detect anomalies in medical images, such as wavelets [2,3], fractal theory [4], statistical methods [5] and most of them used features extracted using image-processing techniques [6]. In addition, some other methods were presented in the literature based on rule based fuzzy [7], Markov models [7], neural networks [9, 10] and fuzzy neural and feature extraction techniques [11]. Most of the computer-aided methods proved to be powerful tools that could assist medical staff in hospitals and lead to better results in diagnosing a patient.

Research into the detection of microcalcifications using the wavelet transform has been carried out in [3]. Given that the microcalcifications correspond to high frequency components of the image spectrum, detection of microcalcifications is achieved by decomposing the mammograms into different frequency sub-bands, suppressing the low-frequency sub-band, and, finally, reconstructing the mammogram from the sub-bands containing only high frequencies. The final images are obtained using sub-band reconstruction. They used two wavelets from Daubechies' family namely the Daubechies' 4 (db 4) filter and Daubechies's 20 (db 20) filter.

According to the theory of deterministic fractal geometry, images can be modeled by deterministic fractal objects which are attractors of sets of two-dimensional (2-D) affine transformations. A methodology based on fractal image modeling is developed to analyze and model breast background structures in [4] and microcalcifications can be enhanced by taking the difference between the original image and the modeled image. This research showed that the fractal modeling method is an effective way to enhance microcalcifications.

Markov random field (MRF) model based method is described in [8]. The detection of tumors is performed in two steps: segmentation and classification. In segmentation, regions of interest are first extracted from the images by adaptive thresholding. A further reliable segmentation is achieved by a MRF model-based method. In classification, the MRF segmented regions are classified into tumor and normal by a fuzzy binary decision tree based on a series of radiographic, density-related features.

A system based on fuzzy-neural and feature extraction techniques for detecting and diagnosing microcalcifications' patterns in digital mammograms is developed in [11]. They investigated and analyzed a number of feature extraction techniques. The following 14 features were used for the proposed method: average histogram, average gray level, energy, modified energy, entropy, and modified entropy, number of pixels, standard deviation, modified standard deviation, skew, modified skew, average boundary gray level, difference and contrast. The formula for entropy, energy, skew, and standard deviation were modified so that the iterations started with the first pixel of the pattern and ended at the final pixel and found that a combination of three features, entropy, 
standard deviation and number of pixels, is the best combination to distinguish a benign microcalcifications pattern from a malignant one. The back-propagation technique was used for classification of features into benign or malignant

Moment based feature descriptors have evolved into a powerful tool for image analysis applications. Geometric moments present a low computational cost, but are highly sensitive to noise. Furthermore reconstruction is extremely difficult. Although not invariant under rotation, Hu's invariants [12] that are derived from geometric moments present invariance under linear transformations. Complex moments provide with additional invariant descriptors, but present the same problems regarding noise and reconstruction.

Global geometric moments and their invariants are widely used in many areas of image analysis, including pattern recognition [12], image reconstruction [13], and fingerprint Recognition [14]. In addition to geometric moments, which are also known as regular or ordinary moments, a number of other moments have been proposed. The notion of complex moments was introduced in [15] for deriving moment invariants. Teague suggested the use of orthogonal moments and introduced complex valued Zernike moments that are defined on a unit disk. A second class of orthogonal moments is given by Legendre moments which make use of Legendre polynomials. The usefulness of Legendre and Zernike moments has been demonstrated, in particular, for image reconstruction [13], [16] and pattern classification [17]. The pseudo-Zernike formulation proposed in [18] further improved these characteristics.

In this class, Legendre and Zernike moments have been extensively researched in the recent past, and several new techniques have emerged involving orthogonal moment based feature detectors [19], [20].

\section{METHODOLOGY}

The block diagram of classification of microcalcification in mammograms based on combined feature set with SVM is shown in Figure 1.

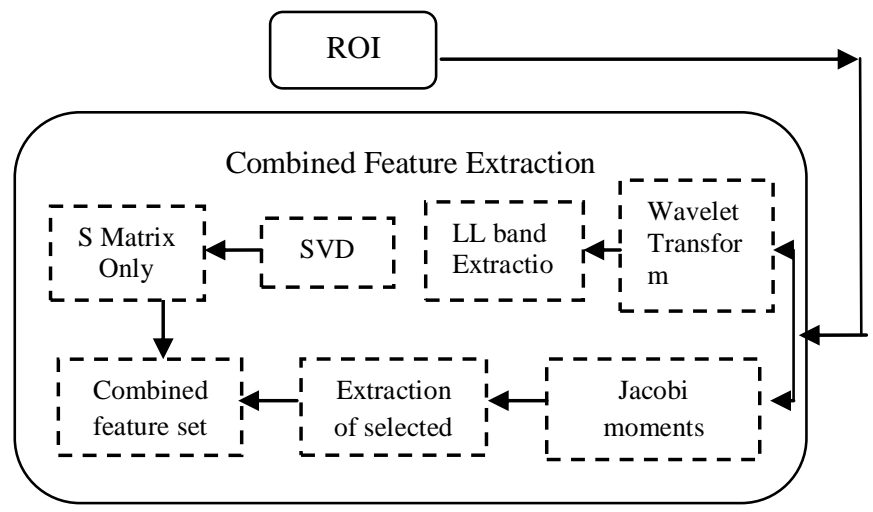

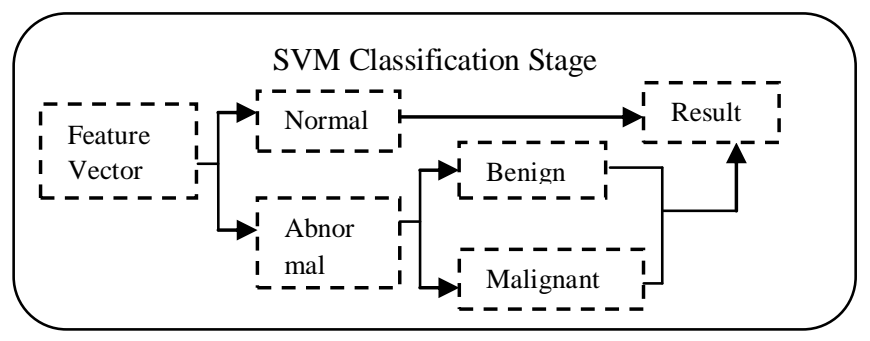

Figure 1: Block diagram of proposed method based on combined feature set with SVM

\subsection{Feature Extraction}

Feature extraction involves simplifying the amount of resources required to describe a large set of data accurately. Analysis with a large number of variables generally requires a large amount of memory and computation power or a classification algorithm which overfits the training sample and generalizes poorly to new samples. Feature extraction is a general term for methods of constructing combinations of the variables to get around these problems while still describing the data with sufficient accuracy.

In the proposed method two features are combined for the classification of micro calcification in the mammogram. They are ' $\mathrm{S}$ ' feature and the Jacobi moments.

\section{1. ' $\mathrm{S}$ ' Feature Extraction.}

Two-dimensional discrete Haar wavelet transform is applied to the given ROI image. It decomposes an input image into four sub-bands, one average component (LL) and three detail components (LH, HL, HH). Then SVD is applied to the LL sub band only.

The Singular Value Decomposition (SVD) is an algebraic technique for factoring any rectangular matrix into the product of three other matrices. Mathematically and historically, it is closely related to Principal Components Analysis (PCA). In addition it provides insight into the geometric interpretation of PCA. As noted previously, the SVD has long been considered fundamental to the understanding of PCA.

The SVD is the factorization of any $k X p$ matrix into three matrices each of which important properties have. That is, any rectangular matrix $\mathrm{A}$ of $\mathrm{k}$ rows by $\mathrm{p}$ columns can be factored in to $\mathrm{U}, \mathrm{S}$ and $\mathrm{V}$.

$$
A=U S V^{T}
$$

Where

- $\quad \mathrm{U}$ is $k X k$ and has columns which are the eigenvectors of $A A^{T}$.

- $\quad \mathrm{V}$ is $\mathrm{p} \times \mathrm{p}$ and has columns which are the eigenvectors of $A^{T} A$. 
- $\quad \mathrm{S}$ is a $k X p$ diagonal matrix with $\mathrm{r}$ non-zero singular values on the diagonal, where $r$ is the rank of $A$. Each singular value is the square root of one of the eigenvalues of both $A A^{T}$ and $A^{T} A$. The singular values are ordered so that the largest singular values are at the top left and the smallest singular values are at the bottom right. that, is $s_{1,1} \geq s_{2,2} \geq s_{3,3}$ etc.

After applying SVD to the LL band of Wavelet Transform, three rectangular matrices $\mathrm{S}, \mathrm{U}$ and $\mathrm{V}$ are obtained. $\mathrm{S}$ is a diagonal matrix which contains the square root Eigen values from $\mathrm{U}$ or $\mathrm{V}$ in descending order is selected and stored it separately in the feature set.

\section{Jacobi Moments Extraction}

The kernel of the Jacobi moments consists of the set of Jacobi polynomials. The Jacobi polynomial of the $n^{\text {th }}$ order, with parameter $\alpha$ and $\beta$, is defined as.

$$
\begin{aligned}
& P_{n}(x ; \alpha, \beta)= \\
& (\alpha+1) \_\mathrm{n} / \mathrm{n} ! \times{ }_{2} F_{1}\left(\begin{array}{c}
-n_{2} n+\alpha+\beta+1 \\
\alpha+1
\end{array} \mid \frac{1-x}{2}\right)
\end{aligned}
$$

where $x \in[-1,1]$. The generalized hyper geometric function, ${ }_{r} F_{s}$, is defined as:

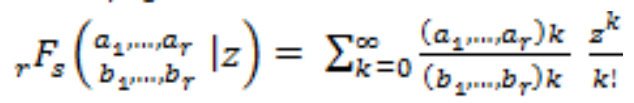

where $\left(a_{1}, \ldots, a_{r}\right) \mathrm{k}=\left(\left(a_{1}\right) k, \ldots,\left(a_{r}\right) k\right.$ and the Pochhammer-symbol:

$$
(a)_{k}=a(a+1)(a+2) \ldots(a+k-1)
$$

with $\mathrm{k}=1,2,3, \ldots$ and $(a)_{0}=1$ and thus have the explicit expression

$$
\begin{aligned}
& P_{n}(x, \alpha, \beta)= \\
& \frac{\Gamma_{(\alpha+n+1)}(\alpha+\alpha+\beta+n+1)}{n ! n=0} \sum_{m=0}^{n}\left(\frac{n}{m}\right) \frac{\Gamma(\alpha+\beta+n+m+m)}{\Gamma(\alpha+m+1)}\left(\frac{z-1}{2}\right)^{m}
\end{aligned}
$$

The Jacobi polynomials can be written in the Rodriguestype formula as:

$$
\begin{aligned}
& (1-x)^{\alpha}(1+x)^{\beta} P_{n}(x ; \alpha, \beta)= \\
& \frac{(-1)^{n}}{2^{n} n !}\left(\frac{d}{d x}\right)^{n}\left[(1-x)^{n+\alpha}(1+x)^{n+\beta}\right]
\end{aligned}
$$

For $\alpha>-1, \beta>-1$, the set of Jacobi polynomials satisfy the orthogonality condition:

$$
\begin{aligned}
& \int_{-1}^{1} \omega(x ; \alpha, \beta) P_{m}(x ; \alpha, \beta) P_{n}(x ; \alpha, \beta) d x= \\
& \rho(n ; \alpha, \beta) \delta_{m n}
\end{aligned}
$$

Where $\delta_{m n}$ is the Kronecker delta and

$$
\omega(x ; \alpha, \beta)=(1-x)^{\alpha}(1+x)^{\beta}{ }_{3}
$$

$\rho(n ; \alpha, \beta)=\frac{{ }_{2}^{\alpha+\beta+1}}{n_{n}+\alpha+\beta+1} \times \frac{\Gamma(n+\alpha+\beta+1) \Gamma(n+\beta+1)}{\Gamma(n+\alpha+\beta+1) n !}$

Where $\Gamma($.$) being the gamma function.$

The Jacobi moment of order $(p+q)$ of an image $\mathrm{f}(\mathrm{x}, \mathrm{y})$ with $\mathrm{M} \mathrm{x} N$ pixels is defined as:

$I_{m n}=\sum_{x=0}^{M-1} \sum_{y=0}^{N-1} f(x, y) \bar{P}_{m}(x) \bar{P}_{n}(x)$

where

$$
\begin{aligned}
& \bar{P}_{m}(x) \equiv \bar{P}_{m}\left(x ; \alpha_{1}, \beta_{1}\right), \\
& \bar{P}_{n}(y) \equiv \bar{P}_{n}\left(y ; \alpha_{2}, \beta_{2}\right),
\end{aligned}
$$

and

$$
\bar{P}_{n}(x ; \alpha, \beta)=P_{n}(x ; \alpha, \beta) \sqrt{\frac{\omega\left(\alpha_{j} \alpha_{2} \beta\right)}{\rho\left(n ; \alpha_{2} \beta\right)}}
$$

So that:

$\sum_{x=0}^{N-1} \bar{P}_{m}(x ; \alpha, \beta) \bar{P}_{n}(x ; \alpha, \beta)=\delta_{m n}$

The term $\bar{P}_{n}($.$) is the weighted Jacobi polynomials.$

The Jacobi moments are calculated the given ROI image using equation (8). Then we have to select the 10 moments they are, $(0,0)(1,1)(2,2)(3,3)(4,4)(5,5)(6,6)(7,7)(8,8)$ $(9,9)$ and $(10,10)$. And all the 10 moments are stored combined with the ' $\mathrm{S}$ ' feature. This combined set is used in the classification phase.

\subsection{Classification phase}

In the proposed method SVM is used as a classifier. Support vector machines (SVMs) are a set of related supervised learning methods that analyze data and recognize patterns, used for classification and regression analysis. The standard SVM is a non-probabilistic binary linear classifier, i.e. it predicts, for each given input, which of two possible classes the input is a member of. Since an SVM is a classifier, then given a set of training examples, each marked as belonging to one of two categories, an 
SVM training algorithm builds a model that predicts whether a new example falls into one category or the other. Intuitively, an SVM model is a representation of the examples as points in space, mapped so that the examples of the separate categories are divided by a clear gap that is as wide as possible. New examples are then mapped into that same space and predicted to belong to a category based on which side of the gap they fall on.

Classification phase executes two phases. In the first one, the classifier is applied to classify mammograms into normal and abnormal cases. Then the mammogram is considered abnormal if it contains tumor.(microcalcification). Finally, the abnormal mammogram is classified into malignant or benign in the second stage. In this classification stage, SVM classifier in every phase is trained at specific number of training set in each category.

\section{3. $1^{\text {ST }}$ STAGE CLASSIFIER}

In the first stage classifier is tested for normal or abnormal images based on combined feature set of "S" matrix and the Jacobi moments features. Then the calculated combined feature set of training images is first trained with the SVM classifier and then tested for all the images including training images for the classification. The number of training and testing images for the $1^{\text {st }}$ stage is given below

\begin{tabular}{|l|l|l|}
\hline $\begin{array}{l}\text { Type of } \\
\text { image }\end{array}$ & $\begin{array}{l}\text { No of training } \\
\text { Images }\end{array}$ & $\begin{array}{l}\text { No of Testing } \\
\text { Images }\end{array}$ \\
\hline Normal & 30 & 194 \\
\hline Abnormal & 12 & 23 \\
\hline
\end{tabular}

\section{4. $2^{\mathrm{ND}}$ STAGE CLASSIFIER}

In this stage, the abnormal images from the 1st stage classifier are further classified into Benign or Malignant. The calculated combined feature set of training images is first trained with the SVM classifier and then tested for all the images including training images for the classification of Benign/Malignant. The number of training and testing images for the $2^{\text {nd }}$ stage is given below

\begin{tabular}{|l|l|l|}
\hline $\begin{array}{l}\text { Type of } \\
\text { image }\end{array}$ & $\begin{array}{l}\text { No of training } \\
\text { set }\end{array}$ & $\begin{array}{l}\text { No of Testing } \\
\text { set }\end{array}$ \\
\hline Benign & 6 & 10 \\
\hline Malignant & 6 & 13 \\
\hline
\end{tabular}

\section{EXPERIMENTAL AND RESULTS}

Results are obtained using the combination of SVD feature sets and Jacobi feature sets. As the classification results are show in table below

\begin{tabular}{|l|l|}
\hline Image Type & Classification \\
\hline Normal & 84.06 \\
\hline
\end{tabular}

\begin{tabular}{|l|l|}
\hline Abnormal & 82.6 \\
\hline Benign & 90.0 \\
\hline Malignant & 84.6 \\
\hline
\end{tabular}

\section{CONCLUSION}

Classification of micro calcification in mammograms based on combined feature set is proposed. Our method is capable of classifying the given mammogram into Normal/Abnormal as well as Benign/Malignant in case of Abnormal. Our method is successful in detecting the micro calcification in the mammogram. To assess its clinical utility, it has to be tested on much larger sets of data. Furthermore, a clinically useful system for breast cancer detection must be able to detect the breast cancers, not just micro calcifications. Our future work is to extend the combined feature set for the detection of mass classification.

\section{REFERENCES}

[1] Kristin J. McLoughlin, Philip J. Bones and Nico Karssemeijer, "Noise Equalization for Detection of Microcalcification Clusters in Direct Digital Mammogram Images" IEEE Trans. Medical Imaging, vol. 23 pp. 313-320,March.2004.

[2] Zhongchao and Ryosuke," An approach to image segmentation using multiresolution analysis of Wavelets" International Journal of Pattern Recognition and Artificial Intelligence, 7(6):810-815, 1999

[3] T.Wang and N. Karayiannis. "Detection of micro calcification in digital mammograms using wavelets". IEEE Trans. Medical Imaging, 17(4):498-509, 1998.

[4] H. Li et al. "Fractal modeling and segmentation for the enhancement of microcalcifications in digital mammograms". IEEE Trans. Medical Imaging, 16(6):785-798, 1997.

[5] H. Chan et al. Computerized analysis of mammographic microcalcifications in morphological and feature spaces. Medical Physics, 25(10):2007-2019, 1998.

[6] S. Lai, X. Li, and W. Bischof. On techniques for detecting circumscribed masses in mammograms. IEEE Trans. Medical Imaging, 8(4):377-386, 1989.

[7] D. Brazokovic and M. Neskovic. Mammogram screening using multi resolution-based image segmentation. International Journal of Pattern Recognition and Artificial Intelligence, 7(6):14371460, 1993.

[8] H. Li et al. Marcov random field for tumor detection in digital mammography. IEEE Trans. Medical Imaging, 14(3):565-576, 1995.

[9] I. Christoyianni et al. Fast detection of masses in computer-aided mammography. IEEE Signal Processing Magazine, pages 54-64, Jan 2000. 
[10] A. Dhawan et al. Radial-basis-function-based classification of mammographic microcalcifications using texture features. In Proc. of the 17th Annual International Conference of the IEEE Engineering in Medicine and Biology Society, volume 1, pages 535536, 1995.

[11] Brijesh Verma and John Zakos , "A Computer-Aided Diagnosis System for Digital Mammograms Based on Fuzzy-Neural and Feature Extraction Techniques" IEEE Trans on nformation technology in biomedicine, vol. 5, pp 46-54, March 2001.

[12] M.-K. Hu, "Visual pattern recognition by moment invariants," IRE Trans. Inform. Theory, pp. 179-187, Feb. 1962.

[13] C. Teh and R. Chin, "On image analysis by the methods of moments," IEEE Trans. Pattern Anal. Machine Intell., vol. 10, pp. 496-513, July 1988.

[14] Hasan Abdel Qader,and Syed Al-Haddad,'Fingerprint Recognition using Zernike moments",The International Arab journal of information technology, vol 4, Oct 2007.
[15] Y. Abu-Mostafa and D. Psaltis, "Recognitive aspects of moment invariants," IEEE Trans. Pattern Anal. Machine Intell., vol. PAMI-6, pp. 698-706, Nov. 1984.

[16] S. Liao and M. Pawlak, "On image analysis by moments," IEEE Trans. Pattern Anal. Machine Intell., vol. 18, pp. 254-266, Mar. 1996.

[17] A. Khotanzad and Y. Hong, "Invariant image recognition by zernike moments," IEEE Trans. Pattern Anal. Machine Intell., vol.48, pp. 489-497, May 1990.

[18] Mirosław Pawlak, and Simon X. Liao ,'On the Recovery of a Function on a Circular Domain". IEEE Trans. Pattern Anal. Machine Intell., vol. 12, pp. 2736-2755, Oct 2002.

[19] Pew-Thian Yap and Raveendran paramesram,"Jacobi Moments as Image Features". International Journal of Pattern Recognition and Artificial Intelligence, 7(6):594-597, 2004

[20] Pew-Thian Yap and Seng-Huat Ong," Image Analysis Using Hahn Moments", IEEE Trans. Pattern Anal. Machine Intell., vol. 11, pp. 2057-2062, Nov 2007. 